\title{
Increasing Human Brain Excitability by Transcranial High- Frequency Random Noise Stimulation
}

\author{
Daniella Terney, Leila Chaieb, Vera Moliadze, Andrea Antal, and Walter Paulus \\ Department of Clinical Neurophysiology, Georg-August University, 37075 Göttingen, Germany
}

For $>20$ years, noninvasive transcranial stimulation techniques like repetitive transcranial magnetic stimulation (rTMS) and direct current stimulation (tDCS) have been used to induce neuroplastic-like effects in the human cortex, leading to the activity-dependent modification of synaptic transmission. Here, we introduce a novel method of electrical stimulation: transcranial random noise stimulation (tRNS), whereby a random electrical oscillation spectrum is applied over the motor cortex. tRNS induces consistent excitability increases lasting $60 \mathrm{~min}$ after stimulation. These effects have been observed in 80 subjects through both physiological measures and behavioral tasks. Higher frequencies $(100-640 \mathrm{~Hz})$ appear to be responsible for generating this excitability increase, an effect that may be attributed to the repeated opening of $\mathrm{Na}^{+}$channels. In terms of efficacy tRNS appears to possess at least the same therapeutic potential as rTMS/tDCS in diseases such as depression, while furthermore avoiding the constraint of current flow direction sensitivity characteristic of tDCS.

Key words: transcranial random noise stimulation (tRNS); primary motor cortex (M1); transcranial magnetic stimulation (TMS); serial reaction time task (SRTT); human; neuromodulation

\section{Introduction}

Neuroplasticity is an ongoing, self-organizing, adaptive process widespread in cortical areas; it allows the brain to learn and adapt to new environmental situations. External influences on neuroplastic processes may be used for functional improvement of diseases, in particular for improving cortical functions such as learning. The most well known method currently used to influence excitability of the brain by external means is transcranial magnetic stimulation (TMS) (Barker et al., 1985). It was followed by various repetitive stimulation paradigms, most recently by theta burst stimulation (TBS) (Huang et al., 2005). Although TBS increased the efficacy of rTMS by reducing stimulus intensity and the number of pulses required to achieve similar aftereffects, its upper safety limits are still unclear due to the potential risk of rTMS inducing seizures (Wassermann, 1998).

Another approach, weak transcranial direct current stimulation (tDCS) of the brain has so far avoided this risk. tDCS was investigated intermittently within the last four decades, but entered into neurobiological and clinical plasticity research only after its efficacy for modulating neuroplasticity could be unambiguously quantified by comparing TMS-induced motor-evoked potentials (MEPs) before and after tDCS (Nitsche and Paulus,

Received Sept. 4, 2008; revised 0ct. 16, 2008; accepted Nov. 12, 2008.

This work was initiated and funded by an unrestricted grant given by the Rose Foundation to develop new tools for the treatment of multiple sclerosis patients (D.T., L.C., W.P.) and the Bernstein Center for Computational Neuroscience Göttingen (V.M., A.A., W.P.) (BMBF 01GQ0432). We thank neuroConn for their cooperation and rapid adaptation of the stimulation device to our needs, and Michael Nitsche, Marom Bikson, and Klaus Schellhorn for their helpful comments.

The authors declare no competing financial interests.

Correspondence should be addressed to Andrea Antal, Department of Clinical Neurophysiology, Georg-August University, Robert-Koch-Strasse 40, 37075 Göttingen, Germany. E-mail: aantal@gwdg.de.

DOI:10.1523/JNEUROSCI.4248-08.2008

Copyright $\odot 2008$ Society for Neuroscience ～0270-6474/08/2814147-09\$15.00/0
2000, 2001). When compared with pulsed rTMS, tDCS represents the other end of the stimulation spectrum by delivering continuous electric current, which leads to "brain polarization." tDCS is able to induce long-lasting changes in cortical excitability in a reversible, relatively selective, painless, and safe manner. Generally, motor cortex (M1) excitability is enhanced by anodal and decreased by cathodal stimulation (Nitsche and Paulus, 2000).

Transcranial random noise stimulation (tRNS) of the human brain is a new technique (Fig. 1). Only one study so far has used noisy galvanic stimulation at a very low-frequency $(<2 \mathrm{~Hz})$ range targeting the vestibular nerves of patients with levodoparesponsive and unresponsive parkinsonism over a $24 \mathrm{~h}$ period (Yamamoto et al., 2005) and successfully improving parkinsonian symptoms. In this article, we demonstrate a new method of enhancing corticospinal excitability as measured by TMS, by applying weak tRNS for 10 min over the M1. Furthermore, a behavioral task was used to study tRNS-driven changes in performance during a variant of the serial reaction time task (SRTT) (Nissen and Bullemer, 1987), which is a standard paradigm to test implicit motor learning. In addition, we show how a cognitive or motor activity performed during stimulation can reduce the efficacy of tRNS, as previously described in studies using tDCS (Antal et al., 2007). The repeated potentiation of sodium channels has been suggested to be a putative mechanism of tRNS action; its aftereffects may outlast those observed after tDCS stimulation.

\section{Materials and Methods \\ Subjects}

Altogether, 80 healthy volunteers ( 32 men and 48 women; mean age, $25.74 \pm 5.13$ years; age range, $20-44$ years) were informed about all aspects of the experiments, and all gave informed consent. None of the subjects suffered from any neurological or psychological disorders, had metallic implants/implanted electric devices, or took any medication regularly, and none of them took any medication in the 2 weeks before their 

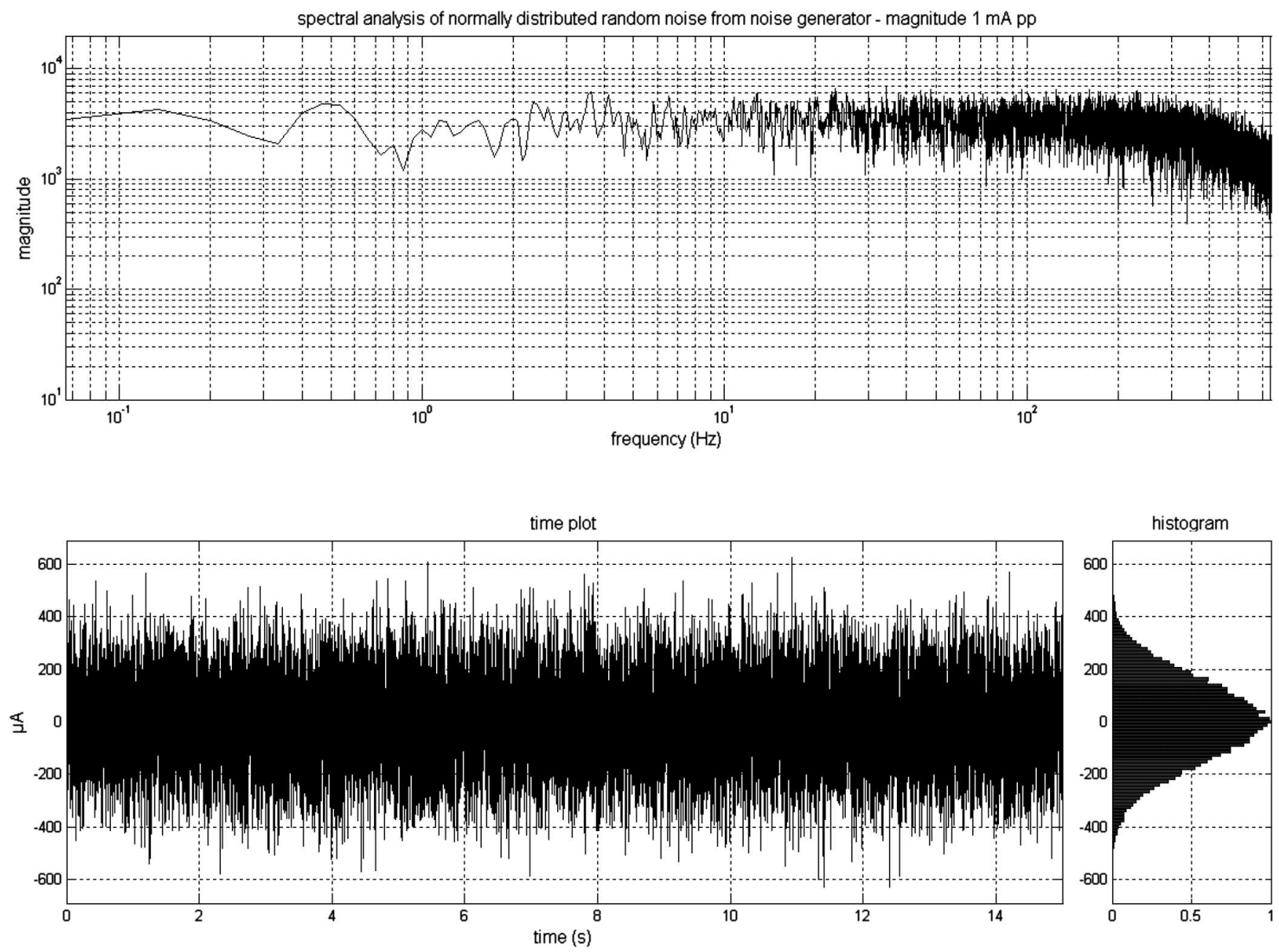

Figure 1. The output signal of DC-Stimulator PLUS, as a frequency distribution of the signal, the time plot of the signal, and a histogram. The signal was generated by a computer. In the stimulation mode "noise," there is a random level of current generated for every sample (sampling rate 1280 samples/s). The random numbers are normally distributed; the probability density function follows a bell-shaped curve. The amplitude of $1 \mathrm{~mA} \mathrm{pp}$ means that $99 \%$ of all generated amplitude values were between $+500 \mu \mathrm{A}$ and $-500 \mu \mathrm{A}$.

participation in any of the experiments. All subjects were right handed, according to the Edinburgh handedness inventory (Oldfield, 1971). We conformed to the Declaration of Helsinki, and the experimental protocol was approved by the Ethics Committee of the University of Göttingen.

Altogether, 47 healthy subjects (motor cortex: 17 participants; 21-27 years old; mean age $=23.71 \pm 2.08 ; 6$ male; low-frequency/high-frequency: 12 participants; $20-28$ years old; mean age $=23.83 \pm 3.28 ; 7$ male; DC-shift-induced excitability changes: 8 participants; $22-38$ years old; mean age $=25 \pm 5.12$; 4 male; premotor cortex: 10 subjects; $22-39$ years old; mean age $=26.5 \pm 6.31 ; 4$ male) participated in the singlepulse TMS study. Ten healthy subjects $(22-44$ years old; mean age $=$ $27.6 \pm 6.67 ; 3$ male) were involved in the paired-pulse TMS experiments, and four subjects participated in both single- and paired-pulse MEP experiments. Seventeen volunteers (22-31 years old; mean age $=25.29 \pm$ $2.89 ; 8$ male) took part in the implicit learning study. Twelve subjects were involved in the task-related modulation study (22-44 years old; mean age $=26.75 \pm 6.08 ; 4$ male). Three subjects participated both in the single-pulse MEP and in the implicit learning experiment. Two subjects were involved in both the single-pulse MEP and task-related modulation experiment.

\section{Random noise stimulation}

Stimulation was delivered by a battery-driven electrical stimulator (Version eldith DC-Stimulator-Plus, neuroConn) through conductiverubber electrodes, placed in two saline-soaked sponges. In the stimulation mode "noise" there is a random level of current generated for every sample (sampling rate $1280 \mathrm{samples/s).} \mathrm{The} \mathrm{random} \mathrm{numbers} \mathrm{are} \mathrm{nor-}$ mally distributed; the probability density function follows a bell-shaped curve. In the frequency spectrum all coefficients have a similar size ("white noise"). The noise signal contains all frequencies up to half of the sampling rate, i.e., a maximum of $640 \mathrm{~Hz}$ (Fig. 1). In a second experiment this frequency spectrum was separated into a low $(0.1-100 \mathrm{~Hz})$ - and high (101-640 Hz)-frequency spectrum. Because of the statistical characteristics, the signal has no DC offset, provided that the offset is set to zero.

The stimulation electrode was placed over the left motor cortex, which was determined by single pulse TMS. During the premotor single-pulse TMS study, the stimulation electrode was placed over the premotor cortex $(2.5 \mathrm{~cm}$ anterior from the motor cortex). To identify the primary motor and premotor cortex the same method was used as that implemented in previous TMS and tDCS studies (e.g., Fink et al., 1997; Münchau et al., 2002). The reference electrode was placed over the contralateral orbit. The size of the stimulation electrode was $4 \times 4 \mathrm{~cm}$ and the reference electrode was $6 \times 14 \mathrm{~cm}$. The electrodes were fixed by elastic bands. tRNS was applied for 10 min with a current strength of $1000 \mu \mathrm{A}$. The maximal current density was $62.5 \mu \mathrm{A} / \mathrm{cm}^{2}$ over the motor cortex, which is below the safety parameters accepted for tDCS (Nitsche et al., 2003). The current density was $11.9 \mu \mathrm{A} / \mathrm{cm}^{2}$ at the reference electrode. For sham stimulation the current was applied for $30 \mathrm{~s}$ at the beginning of the stimulation session, and then turned down. However, the screen on the stimulator did show the remaining time until the end of the stimulation session, as per the verum stimulation condition. Subjects were blinded for stimulation conditions in all of the studies. 


\section{Electrophysiological studies: transcranial magnetic stimulation}

To detect current-driven changes of excitability, motor-evoked potentials (MEPs) of the right first dorsal interosseus muscle (FDI) were recorded following stimulation of its motor-cortical representation field by single-pulse TMS. These were induced using a Magstim 200 magnetic stimulator, with a figure-of-eight standard double magnetic coil (diameter of one winding, $70 \mathrm{~mm}$; peak magnetic field, $2.2 \mathrm{~T}$; average inductance, $16.35 \mu \mathrm{H})$. The coil was connected to two monophasic Magstim 200 stimulators via a bistim module during the paired-pulse TMS study. Surface electromyogram (EMG) was recorded from the right FDI through a pair of $\mathrm{Ag}-\mathrm{AgCl}$ surface electrodes in a belly-tendon montage. Raw signals were amplified, bandpass filtered (2 $\mathrm{Hz}$ to $3 \mathrm{kHz}$; sampling rate, $5 \mathrm{kHz}$ ), digitized with a micro $1401 \mathrm{AD}$ converter (Cambridge Electronic Design) controlled by Signal Software (Cambridge Electronic Design, version 2.13), and stored on a personal computer for off-line analysis. Complete relaxation was controlled through auditory and visual feedback of EMG activity and whenever it was necessary, the subject was instructed to relax. The coil was held tangentially to the skull, with the handle pointing backwards and laterally at $45^{\circ}$ from the midline, resulting in a posterior-anterior direction of current flow in the brain. This orientation of the induced electrical field is thought to be optimal for predominantly transsynaptic mode of activation of corticospinal system. The optimum position was defined as the site where TMS resulted consistently in the largest MEP in the resting muscle. The site was marked with a skin marker to ensure that the coil was held in the correct position throughout the experiment.

\section{Experimental design}

Subjects were seated in a comfortable reclining chair with a mounted headrest throughout the experiments. Within each type of experimental session, the measurements were always performed by the same investigator.

\section{Single-pulse TMS}

Motor cortex stimulation. Seventeen subjects participated in two experimental sessions, on separate days, at least $3 \mathrm{~d}$ apart to avoid carryover effects. The subjects received $\mathrm{RN}$ and sham stimulation in a randomized order. Resting motor threshold (RMT), active motor threshold (AMT), the intensity to evoke MEP of $\sim 1 \mathrm{mV}$ peak-to-peak amplitude (SI1mV), and a baseline of TMS-evoked MEPs (40 stimuli) were recorded at 0.25 $\mathrm{Hz}$ before the stimulation.

Stimulus intensities (in percentage of maximal stimulator output) of TMS were determined at the beginning of each experiment. RMT was defined as the minimal output of the stimulator that induced a reliable MEP $(\sim 50 \mu \mathrm{V}$ in amplitude) in at least three of six consecutive trials when the FDI muscle was completely relaxed. AMT was defined as the lowest stimulus intensity at which three of six consecutive stimuli elicited reliable $\mathrm{MEP}(\sim 200 \mu \mathrm{V}$ in amplitude) in the tonically contracting FDI muscle (Rothwell et al., 1999).

Following stimulation, 40 single test-pulse MEPs were recorded at 0.25 $\mathrm{Hz}$, i.e., $\sim 0,5$, and $10 \mathrm{~min}$ after stimulation and then every $10 \mathrm{~min}$ up to 60 min.

Additionally, eight subjects underwent the same single-pulse TMS experiment (as described previously) to investigate the length of the aftereffect of the stimulation. Subjects were measured 0, 5, and $10 \mathrm{~min}$ after stimulation, then every $10 \mathrm{~min}$ up to $60 \mathrm{~min}$, then twice in the second hour, then 4,6 , and $24 \mathrm{~h}$ after stimulation. Both active and sham stimulation conditions were applied.

In a second sham-controlled experiment, the random noise frequency was divided into a low $(0.1-100 \mathrm{~Hz})$ - and high $(101-640 \mathrm{~Hz})$-frequency spectrum. Twelve participants underwent the same protocol as previously described.

To conclusively exclude DC-shift-induced excitability changes, eight subjects underwent the same protocol as previously described, in which the standard DC electrode montage was used (active electrode, anodal; reference electrode, cathodal) and then the electrode montage was reversed (cathodal-anodal).

Premotor cortex stimulation. Ten subjects participated in two experi- mental sessions on separate days, at least $3 \mathrm{~d}$ apart to avoid carryover effects. The subjects received tRNS and sham stimulation in a randomized order. The study protocol was performed as previously described.

\section{Paired-pulse TMS}

TMS measurements included RMT, AMT, and SI $1 \mathrm{mV}$, short-interval intracortical inhibition (SICI)/intracortical facilitation (ICF), longinterval intracortical inhibition (LICI), recruitment curves, and cortical silent period (CSP).

Ten subjects participated in four experimental sessions [(1) tRNS: recruitment curves and SICI/ICF; (2) tRNS: LICI and CSP; (3) sham: recruitment curves and SICI/ICF; and (4) sham: LICI and CSP] on separate days at least $3 \mathrm{~d}$ apart to avoid carryover effects. The subjects received RN and sham stimulation in a randomized order. Stimulus intensities (in percentage of maximal stimulator output) of TMS were determined at the beginning of each experiment. SI $1 \mathrm{mV}$ was determined with single-pulse TMS first (the amplitude of the test MEP was matched before and after tRNS). RMT and AMT were defined as previously mentioned.

SICI/ICF and LICI were measured with two different protocols of single- and paired-pulse TMS applied in a random order at $0.25 \mathrm{~Hz}$. For SICI/ICF, two magnetic stimuli were given through the same stimulating coil, and the effect of the first (conditioning) stimulus on the second (test) stimulus was investigated (Kujirai et al., 1993). To avoid any floor or ceiling effect, the intensity of the conditioning stimulus was set to a relatively low value of $80 \%$ of AMT. The test-stimulus intensity was adjusted to SI $1 \mathrm{mV}$. SICI was measured with interstimulus intervals (ISI) of 2 and $4 \mathrm{~ms}$, and ICF with ISIs of 9, 12, 15, and $25 \mathrm{~ms}$. The control condition (test pulse alone) was tested 40 times, and each of the conditioning-test stimuli 20 times. The mean peak-to-peak amplitude of the conditioned MEP at each ISI was expressed as a percentage of the mean peak-to-peak size of the unconditioned test pulse. The second protocol tested LICI with two suprathreshold stimuli applied with ISIs of 50, 100, 150, and $200 \mathrm{~ms}$ (Valls-Solé et al., 1992). The intensity of both stimuli was set to $110 \%$ of RMT. Here as well, the intensity was set to this relatively low value to avoid any floor or ceiling effect. The control condition (first pulse alone) was tested 40 times, whereas each of the paired stimuli was tested 20 times. LICI was taken as the mean percentage inhibition of conditioned MEP at ISIs of 50, 100, 150, and $200 \mathrm{~ms}$.

Recruitment curves were measured with three different and increasing stimulus intensities $(110 \%, 130 \%$, and $150 \%$ of RMT), each with 10 pulses. A mean was calculated for all intensities. Finally, 10 pulses with SI $1 \mathrm{mV}$ and 10 pulses with $120 \%$ RMT were applied under tonic contraction of the right first dorsal interosseus muscle. CSPs were separately determined, in rectified and averaged EMG traces with a prestimulus period of $100 \mathrm{~ms}$. CSP (in ms) was measured from the TMS stimulus to the point where the signal reached the amplitude of the mean prestimulus EMG activity again for $>5 \mathrm{~ms}$.

\section{Behaviorial studies}

\section{SRTT}

Subjects were seated in front of a computer screen at eye level behind a response pad with four buttons numbered 1-4 and were instructed to push each button with a different finger of the right hand (index finger for button 1, middle finger for button 2, ring finger for button 3, and little finger for button 4). An asterisk appeared in one of four positions that were horizontally spaced on a computer screen and permanently marked by dots. The subjects were instructed to press the key corresponding to the position of the asterisk as fast as possible. After a button was pushed, the go signal disappeared. The next go signal was displayed $500 \mathrm{~ms}$ later. The test consisted of eight blocks of 120 trials. In blocks 1 and 6, the sequence of asterisks followed a pseudorandom order in that asterisks were presented equally frequently in each position and never in the same position in two subsequent trials. In blocks $2-5,7$, and 8 , the same 12 trial sequence of asterisk positions repeated itself 10 times (abadbcdacbdc). Subjects were not informed about the repeating sequence.

In six subjects, the first three blocks of the previously used test were repeated 1 (block 9: pseudorandom; blocks 10-11: repeated sequences) and 2 h (block 12: pseudorandom; blocks 13-14: repeated sequences) 
after stimulation. Differences in performance between blocks 9-10 and 12-13 also represent a measure of implicit learning. In the SRTT study, the current was delivered during the blocks $2-5$, which lasted $\sim 7 \mathrm{~min}$. The order of verum and sham stimulation was randomized. The current was always ramped up or down over the first and last $2 \mathrm{~s}$ of stimulation.

\section{Task-related modulation of $t R N S$}

The three experimental sessions were conducted in a repeatedmeasurement design using a randomized order, with a break of at least $3 \mathrm{~d}$ between each session. First, the left motor-cortical representational field of the right FDI was identified using TMS. After determining the resting and active motor thresholds, a baseline of TMS-evoked MEPs (25 stimuli) was recorded at $0.25 \mathrm{~Hz}$. Afterward, one stimulation electrode was fixed at the representational field of the right FDI, and the other was fixed at the contralateral forehead above the orbita.

During tRNS, subjects were passively sitting during the stimulation (experiment 1), had their attention directed toward a cognitive test (experiment 2) or were instructed to push a ball in their right hand (experiment 3). After termination of RNS, 25 MEPs were recorded every fifth minute up to $30 \mathrm{~min}$ and then every $15 \mathrm{~min}$ up to $2 \mathrm{~h}$.

During the stimulation in experiment 2, the subjects were required to fill out a cognitive test that was presented on a computer monitor. The subjects had to push a suitable button with their right index finger to give the correct answer. The test was presented in German and downloaded from a commercial intelligence test homepage. The questions were on a variety of subjects. In experiment 3 , the subjects were instructed to push a ball $(8 \mathrm{~cm}$ diameter) in their right hand. The ball was connected to a display where the actual values related to pressure were quantified. Before the stimulation session, the subjects were asked to push the ball as hard as possible. During the tRNS session, subjects had to push the ball to half-maximal contraction as previously shown.

\section{Safety}

\section{Neuron-specific enolase determination}

To assess the safety of tRNS, we measured serum neuron-specific enolase (NSE), a sensitive marker of neuronal damage, evident in many neurological disorders, e.g., in epilepsy (Steinhoff et al., 1999). Elevated NSE concentration is a specific marker in intractable temporal lobe epilepsy. A blood sample for NSE-measurement was taken in six healthy subjects before and $10 \mathrm{~min}$ after stimulation. Furthermore, in one subject, who was stimulated for 8 consecutive days, this measurement was done on every day.

\section{EEG recording}

The EEG was recorded using a three-channel montage. One electrode was placed over $\mathrm{Oz}$ and two laterally above the motor region (C3 and C4) in accordance with the international 10/20 system. The impedance was kept at $<5 \mathrm{k} \Omega$. Linked mastoids (RLm) were used as a reference. The ground electrode was positioned on the forehead. Data were collected with a sampling rate of $1000 \mathrm{~Hz}$ using BrainAmp system (Brain Products) and were analyzed off-line (Brain Vision Analyzer, Brain Products).

The EEG experiments were conducted in a repeated-measurement design (tRNS and sham) using a randomized order, with a minimum break of $1 \mathrm{~d}$ between each stimulation session. Two minutes EEG was recorded at rest before and three times after stimulation (immediately and 7 and $14 \mathrm{~min}$ after the end of the stimulation). EEG epochs (2 min) were segmented for $30 \mathrm{~s}$ and filtered by using $0.1 \mathrm{~Hz}$ ( $24 \mathrm{~dB} /$ octave) low cutoff and a $70 \mathrm{~Hz}$ ( $24 \mathrm{~dB} /$ octave) high cutoff and $50 \mathrm{~Hz}$ notch filters. In addition to semiautomatic artifact detection (200 $\mu \mathrm{V}$ amplitude criterion), all epochs were visually inspected, and those containing eye blinks or muscle movement artifacts were excluded. After artifact rejection, all of the epochs were segmented into $2 \mathrm{~s}$, and fast Fourier transformation (FFT) was calculated for all electrodes $(0.5 \mathrm{~Hz}$ resolution, and $10 \% \mathrm{Ham}$ ming window). The FFT segments were averaged for each $30 \mathrm{~s}$. The mean activity in voltage was calculated and exported from each frequency bands (theta band $4.5-7 \mathrm{~Hz}$, alpha band $8-12 \mathrm{~Hz}$, beta band $12.5-30 \mathrm{~Hz}$, and gamma band $31-49 \mathrm{~Hz}$ ) for statistical analysis.

For sham stimulation, the current was turned on for $30 \mathrm{~s}$ at the begin- ning of the stimulation. Subjects were blinded for stimulation conditions in all of the studies.

\section{Data analyses}

\section{Electrophysiological studies}

Single-pulse TMS. Repeated measurements of ANOVAs [condition $($ tRNS vs sham) $\times$ time (before; $0,5,10,20,30,40,50,60$ min after stimulation; ( $n=8$ : before; $0,5,10,20,30,40,50,60,90 \mathrm{~min}$ and 2, 4, 6, $24 \mathrm{~h}$ after stimulation)] were used to compare the different conditions. Effects were considered significant if $p<0.05$. In the case of a significant interaction of time and stimulation condition, a Tukey's post hoc test was performed. Student's $t$ test was used to compare the motor thresholds (RMT, AMT, and SI $1 \mathrm{mV}$ ) between experimental sessions. All data are given as means \pm SEM.

Paired-pulse TMS. For each measurement (SI1mV, RMT, AMT, SICI, ICF, LICI, and CSP), we performed separate ANOVAs for repeated measurements by using the mean values from each subject as the dependent variable. In addition to the factor "stimulation type" (tRNS vs sham), the ANOVA model included the factor "ISI" (2, 4, 7, 9, 12, 15, and $25 \mathrm{~ms}$ ) when SICI and ICF was analyzed, the factor "intensity" (100\%, 130\%, and $150 \%$ of RMT) for recruitment curves, or the factor "intensity" $(120 \%$ RMT and SI $1 \mathrm{mV}$ ) for CSP. A $p$ value of $<0.05$ was considered significant for all statistical analyses. In the case of a significant interaction between ISI/intensity and stimulation condition, a Tukey post hoc test was performed. Student's $t$ test was used to compare the motor thresholds (RMT, AMT, and SI $1 \mathrm{mV}$ ) between experimental sessions. Data are expressed as mean \pm SEM.

\section{Behavioral studies}

SRTT analysis. Concerning the implicit learning paradigm, statistical analysis was performed with repetitive-measures ANOVA (independent variables current condition and block) for reaction time (RT), error rate (ER), and variability. As the RT and ER differences between blocks 5 and 6 are thought to represent an exclusive measure of implicit learning, interactive Student's $t$ tests were performed to compare the respective differences between tRNS and sham conditions. In each trial, RT was measured from the appearance of the "go" signal until the first button was pushed by the subject. For each block of trials of a given experimental condition, mean RT was calculated for each subject separately. Furthermore, as a measure of the variability of the RTs, we have calculated the coefficient of variation (the ratio of the SD to the mean $\times 100$ ). An ER was calculated to assess the number of incorrect responses for each block and each subject in each stimulation condition.

Task-related modulation of $t R N S$. Repeated-measures ANOVA [experiment (passive vs cognitive/motor) $\times$ time (before and 5, 10, 15, 20, 25, and $30 \mathrm{~min}$ after stimulation, then every $15 \mathrm{~min}$ up to $2 \mathrm{~h}$ )] was used to compare different task conditions during tRNS. Effects were considered significant if $p<0.05$. In case of the significant interaction of time and stimulation condition, a Tukey post hoc test was performed. Student's $t$ test was used to compare the motor thresholds (RMT, AMT, and SI $1 \mathrm{mV}$ ) between experimental sessions. All data are given as means + SEM.

\section{Safety}

NSE determination. Two-tailed $t$ tests (paired samples, critical $p$ value 0.05 ) were performed to compare NSE values before and after tRNS.

EEG recording. To compare the effect of stimulation on the EEG spectrum, a repeated-measures ANOVA (independent variable: tRNS vs sham $\times$ time points after stimulation; dependent variable: FFT power in a given frequency band) was calculated.

\section{Results}

All of the subjects tolerated the stimulation; none of the experimental sessions were interrupted due to side effects of the stimulation. Only two of 80 subjects reported a slight burning sensation under the electrodes during the stimulation. 
Table 1. Results of the statistical analyses in the case of the single- and paired-pulse TMS studies over the primary motor cortex

\begin{tabular}{|c|c|c|c|c|c|}
\hline & Measurement & Factor & df & $F / t^{a}$ & $p$ \\
\hline \multicolumn{6}{|l|}{ Single-pulse TMS } \\
\hline \multirow[t]{3}{*}{ Student's $t$ test } & RMT & & 10 & 0.90 & 0.39 \\
\hline & AMT & & 10 & 1.68 & 0.12 \\
\hline & $\mathrm{SI} 1 \mathrm{mV}$ & & 10 & 0.42 & 0.69 \\
\hline \multirow[t]{3}{*}{ ANOVA } & & Condition & 1 & 7.24 & 0.01 \\
\hline & & Time & 28 & 4.01 & $<0.01$ \\
\hline & & Condition $\times$ time & 28 & 3.53 & $<0.01$ \\
\hline \multicolumn{6}{|l|}{ Paired-pulse TMS } \\
\hline \multirow[t]{3}{*}{ Student's $t$ test } & RMT & & 9 & 0.42 & 0.68 \\
\hline & AMT & & 9 & 0.90 & 0.39 \\
\hline & $\mathrm{SI} 1 \mathrm{mV}$ & & 9 & 0.01 & 1.00 \\
\hline \multirow{15}{*}{ ANOVA } & & Condition & 1 & 0.80 & 0.39 \\
\hline & & Intensity & 2 & 19.03 & $<0.01$ \\
\hline & RECR & Condition $\times$ intensity & 2 & 0.38 & 0.69 \\
\hline & & Condition & 1 & 0.38 & 0.54 \\
\hline & & $|S|$ & 1 & 47.94 & $<0.01$ \\
\hline & $\mathrm{SICl}$ & Condition $\times|S|$ & 1 & 0.13 & 0.73 \\
\hline & & Condition & 1 & 0.58 & 0.46 \\
\hline & & $|S|$ & 3 & 0.88 & 0.46 \\
\hline & ICF & Condition $\times|S|$ & 3 & 5.56 & $<0.01$ \\
\hline & & Condition & 1 & 0.23 & 0.64 \\
\hline & & ISI & 4 & 4.04 & 0.01 \\
\hline & $\mathrm{LICl}$ & Condition $\times|S|$ & 4 & 0.37 & 0.83 \\
\hline & & Condition & 1 & 0.63 & 0.44 \\
\hline & & Intensity & 1 & 1.05 & 0.33 \\
\hline & CSP & Condition $\times$ intensity & 1 & 0.81 & 0.38 \\
\hline
\end{tabular}

RECR, Recruitment curves. Bold indicates significant values. ${ }^{a}$ for ANOVA and $t$ for Student's $t$ test.

\section{Electrophysiological studies: MEPs}

Single-pulse TMS

When 10 min tRNS was applied over the primary motor cortex, the induced excitability increases rose up to $20-50 \%$, as revealed by TMS. They last for $60 \mathrm{~min}$ after stimulation. Repeated measurements of ANOVA revealed a significant main effect of condition $\left(F_{(1,28)}=7.24, p=0.01\right)$ and time $\left(F_{(8,224)}=4.01, p<\right.$ $0.001)$ in the case of motor cortex stimulation. The interaction between condition and time was also significant $\left(F_{(8,224)}=3.53\right.$, $p<0.001$ ) (Table 1). According to the post hoc analysis, significantly increased MEPs were observed at the 5 and 10-60 min time points compared with the time point before $(p<0.05)$ tRNS (Fig. 2).

RMT, AMT, and SI1mV baseline values were compared between RN and sham stimulation conditions using Student's $t$ test. There was no significant difference between tRNS and sham stimulation in any of the measurements (Table 1).

Furthermore, we have separated the stimulation spectrum into low (0.1-100 Hz)- and high (101-640 Hz)-frequency ranges. High-frequency stimulation was more effective with regard to changing the level of cortical excitability. Repeated measurements of ANOVA revealed a significant effect of condition $\left(F_{(1,21)}=4.2, p=0.05\right)$ when the high-frequency spectrum stimulation was used, compared with the sham condition. However, there was no significant effect of condition, when the lowfrequency spectrum was applied $\left(F_{(1,20)}=2.22, p=0.15\right)$. There was no significant condition $\times$ time interaction $\left(F_{(7,147)}=1.62\right.$, $p=0.13$ and $F_{(7,140)}=0.78, p=0.61$, respectively) (Fig. 3).

We did not observe any changes in corticospinal excitability when the premotor cortex was stimulated, implying that the effect of tRNS over the M1 is indeed focal. Repeated measurements of ANOVA revealed no significant effect on condition $\left(F_{(1,18)}=\right.$ $0.01, p=0.99)$ nor time $\left(F_{(8,14)}=0.78, p=0.61\right)$. There was no significant condition $\times$ time interaction $\left(F_{(8,14)}=0.69, p=0.70\right)$.

The possibility of a hidden DC shift in the stimulation spectrum as a cause of the excitability increase was excluded by the results of a control experiment conducted by reversing the connection of the electrodes to the stimulator. In the case of measuring DC-shift-induced excitability changes, repeated measurements of ANOVA revealed no significant effect of condition $\left(F_{(1,14)}=0.29, p=0.60\right)$. The effect of time was significant $\left(F_{(8,112)}=\right.$ $2.13, p=0.04)$. There was no significant condition $\times$ time interaction $\left(F_{(8,112)}=\right.$ $0.24, p=0.98$ ).

\section{Paired-pulse TMS}

In our paired-pulse TMS study, we have observed an increase in ICF after tRNS over M1. Repeated measurements of ANOVA revealed no significant effect of condition $\left(F_{(1,9)}=0.58, p=0.46\right)$ or ISI $\left(F_{(3,27)}=0.88, p=0.46\right)$. However, the interaction between condition and ISI was significant $\left(F_{(3,27)}=5.56, p=0.004\right)$. According to the post hoc analysis, significantly increased MEPs were observed at ICF of 12 and $15 \mathrm{~ms}$ after tRNS compared with the sham condition $(p<0.05)$.

tRNS administration had no effect on SICI, LICI, CSP, or motor-evoked recruitment curves as revealed by repeated measurements of ANOVA (Table 1).

\section{Behavioral studies}

SRTT

With regard to the functional effect of tRNS, it significantly improved performance in the acquisition and early consolidation phase of motor learning. This was primarily represented by the differences between blocks 5 and 6 between tRNS and sham conditions, which are exclusive measurements of implicit learning. Compared with the sham stimulation condition, RTs in the SRTT shortened during tRNS of the primary motor cortex; and subjects became faster during the course of the experiment.

Repeated-measures ANOVA revealed a significant effect on blocks $\left(F_{(7,112)}=37.59, p<0.001\right)$. This was caused by an interaction of tRNS versus sham stimulation for block 5 and block 6 , due to a greater difference in the case of $\operatorname{tRNS}(t=-2.87, \mathrm{df}=16$, $p=0.01)$ as revealed by Student's $t$ tests. There was no significant effect on stimulation. However, the stimulation $\times$ blocks interaction was marginally significant $\left(F_{(7,112)}=1.95, p=0.06\right)$. Figure 4 shows the differences between RN and sham stimulation. The paradigm was repeated in six subjects after 1 and $2 \mathrm{~h}$ after stimulation. At these time points the RTs were not significantly different between the tRNS and sham stimulation conditions (see Fig. 4). However, the RTs of the sham and tRNS trials were not the same as those observed after the familiar blocks immediately after stimulation, but are the same after $1 \mathrm{~h}$; the control RTs decreased substantially in the $1 \mathrm{~h}$ period for the familiar block, which may represent consolidation of learning, whereas this was not the case for the tRNS group. Nevertheless, only six subjects were analyzed after $1 \mathrm{~h}$.

For the ER, the ANOVAs showed a significant main effect on 
blocks $\left(F_{(7,112)}=2.54, p=0.02\right)$. Despite this, the results of all other tests remained insignificant. Student's $t$ tests revealed no significant difference between blocks 5 and 6. For RT variability, the ANOVAs showed a significant main effect on blocks $\left(F_{(7,112)}\right.$ $=29,12, p<0.0001)$ without significant interaction between condition and blocks.

\section{Task-related modulation of $t R N S$}

Excitability increase induced by tRNS was modified by paying attention to a task involving mental activity and by contraction of the target muscle during the stimulation. Following tRNS, the amplitude of the MEPs was increased in the passive condition, slightly decreased in the cognitive condition and markedly reduced in the motor condition. When the amplitude of the MEPs was compared with regard to the passive condition and cognitive task before and after stimulation, repeatedmeasures ANOVA revealed a main effect of experiment $\left(F_{(1,11)}=5.45, p=0.04\right)$, but time $\left(F_{(12,132)}=0.50, p=0.91\right)$ was not significant. The interaction between the experiment and time was significant $\left(F_{(12,132)}=2.36, p=0.009\right)$. The post hoc test revealed that, after $t R N S$ in the passive condition, significantly increased MEP amplitudes were observed up to $20 \mathrm{~min}$, and at the 1 and $2 \mathrm{~h}$ time points when compared with the cognitive task condition $(p<0.01)$. When the amplitude of the MEPs was compared with the passive condition and motor task, repeated measures of ANOVA revealed a main effect of experiment $\left(F_{(1,11)}=10.05, p=0.009\right)$, but time $\left(F_{(12,132)}=0.74, p=0.71\right)$ was not significant. The interaction between the experiment and time was significant $\left(F_{(12,132)}=3.96, p<0.001\right)$. The post hoc test revealed that, after tRNS in the passive condition, significantly increased MEP amplitudes were observed up to $25 \mathrm{~min}$ ( $p<$ 0.01 ) compared with the motor condition.

\section{Safety}

The concentration of serum NSE was unchanged after tRNS. Student's $t$ test showed no significant difference between the before and after stimulation NSE concentrations of six healthy subjects $(t=$ $0.09, p=0.93$, mean value before stimulation: $6.96 \pm 1.84 \mu \mathrm{g} / \mathrm{L}$, after stimulation: $6.91 \pm 1.7 \mu \mathrm{g} / \mathrm{L})$. One subject was stimulated for $10 \mathrm{~min}$ every day for 8 consecutive days. The NSE values did not change significantly over the period from the first to last day of stimulation $(t=-0.2, p=$ 0.87 , mean value before stimulation: $9.57 \pm 2.2 \mu \mathrm{g} / \mathrm{L}$, after stimulation: $9.53 \pm 3.0 \mu \mathrm{g} / \mathrm{L})$.

Furthermore, we recorded EEGs before and after tRNS and at baseline.

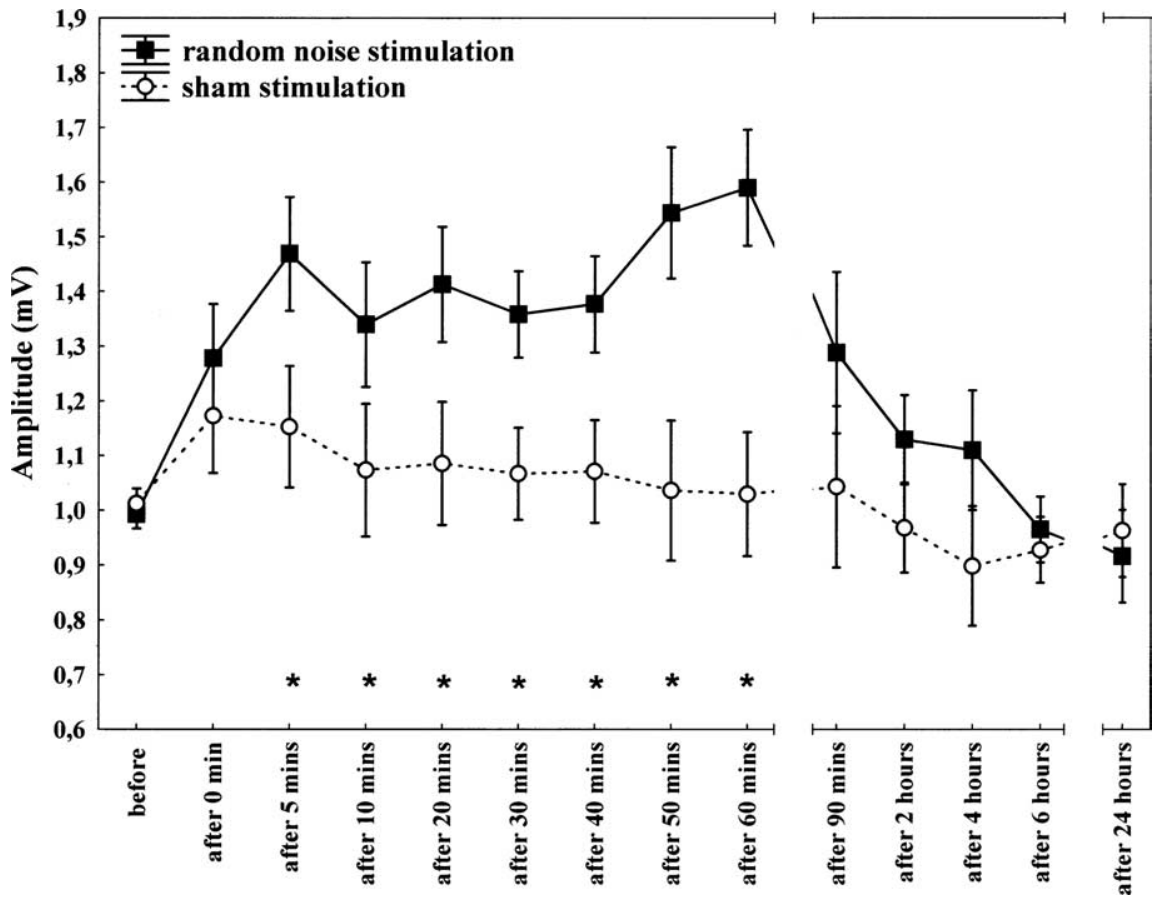

Figure 2. Effect of $10 \mathrm{~min}$ RN stimulation on motor-evoked potentials. Time course of motor cortex excitability changes lasting for 60 min after stimulation, shown after $10 \mathrm{~min}$ RN stimulation over M1 at $1 \mathrm{~mA}$ compared with sham stimulation. The figure shows mean amplitudes and their SEMs up to $60 \mathrm{~min}$ (including all subjects, $n=17$ ) and between $90 \mathrm{~min}$ and $24 \mathrm{~h}$ (including 8 subjects). Asterisks indicate significant differences between MEP amplitudes after 5 and $10-60$ min after stimulation and those

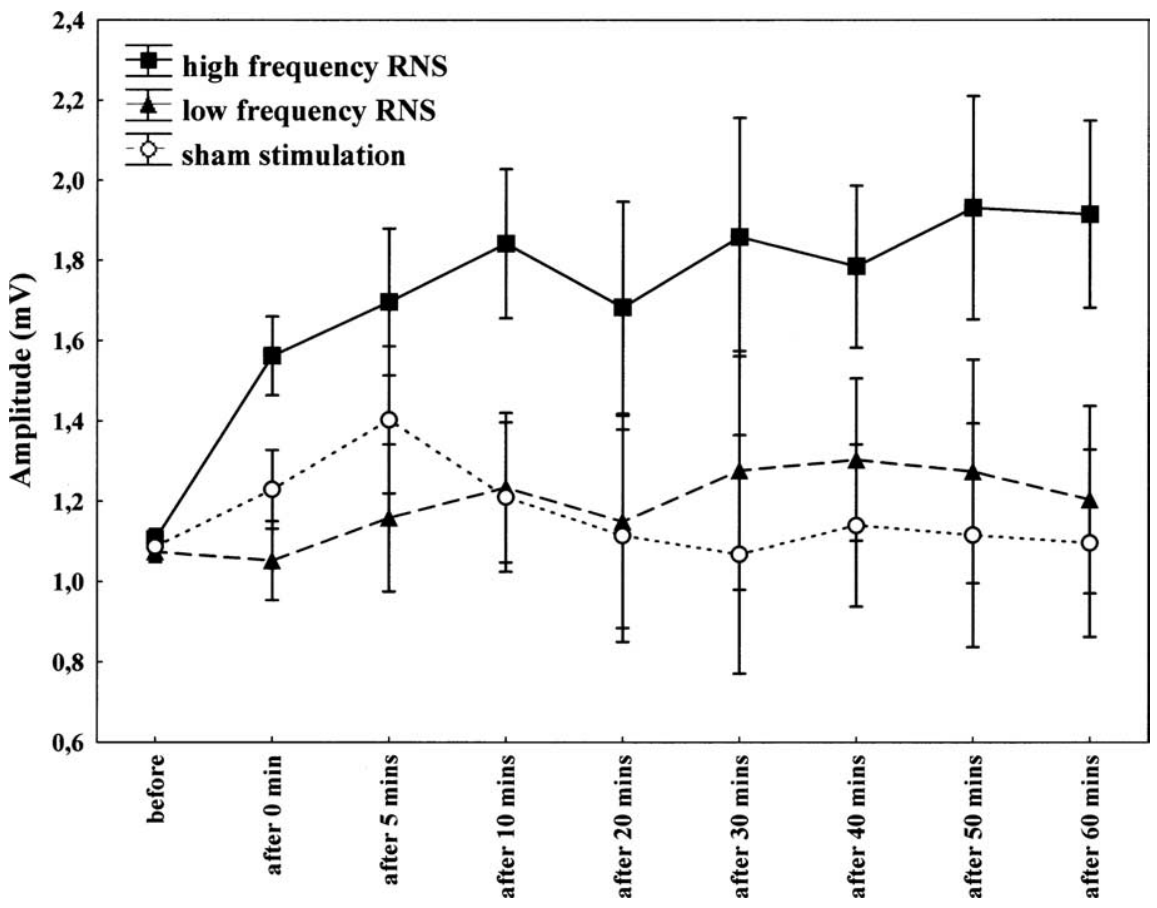

Figure 3. Effect of 10 min of low $(0.1-100 \mathrm{~Hz})$ - and high $(101-640 \mathrm{~Hz})$-frequency RN stimulation on motor-evoked potentials. Time course of motor cortex excitability changes lasting for $60 \mathrm{~min}$ after stimulation, shown after 10 min of high-frequency RN stimulation over M1 at $1 \mathrm{~mA}$ compared with low-frequency and sham stimulation. The figure shows mean amplitudes and their SEMs up to $60 \mathrm{~min}$ (including all subjects, $n=12$ ).

did not find any significant difference regarding any of the frequency bands. Repeated-measures ANOVA revealed no significant interactions between current conditions, time, or channels for any of the different frequencies applied (see supplemental 

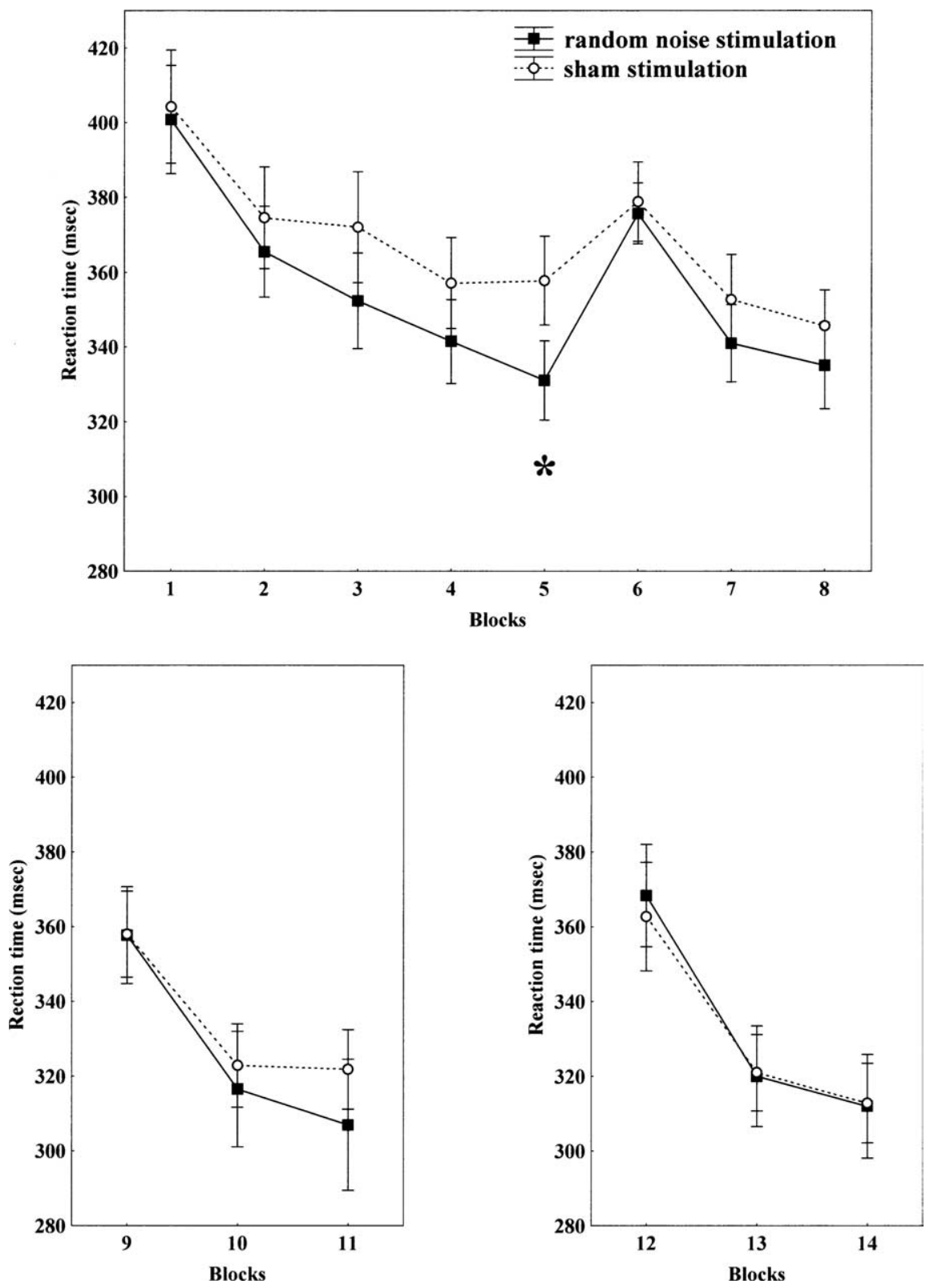

Figure 4. tRNS of the primary motor cortex improves implicit motor learning in its early phase. Reaction times decrease faster in the tRNS condition when compared with the sham stimulation condition (top). Moreover, the RT difference comparing blocks 5 and 6, which indicates implicit sequence learning, is bigger for the tRNS condition, when compared with sham condition. The asterisk indicates a significant difference regarding reaction time differences between blocks 5 and 6 between RN and sham stimulation. In 1 and $2 \mathrm{~h}$ after stimulation, this significant difference was no longer detectable (bottom panels).

Table 2, available at www.jneurosci.org as supplemental material). Additionally, we did not see any abnormal EEG activity after tRNS. Therefore, we can conclude that limited exposure to tRNS of the cortex using the parameters we applied here is safe.

\section{Discussion}

In this study, we demonstrate that weak tRNS over M1 enhances corticospinal excitability both during and after stimulation in the healthy human brain. Furthermore, our results suggest that the high-frequency subdivision of the whole tRNS spectrum between 100 and $640 \mathrm{~Hz}$ is functionally responsible for inducing excitability in the M1. In terms of commonly used noninvasive excitability parameters, we found an increased ICF after tRNS over M1 using the paired-pulse paradigm. tRNS application had no effect on
SICI, LICI, CSP, or motor-evoked recruitment curves [for an overview of methods used to study the modulation of human motor cortex excitability in local circuits, see Paulus et al. (2008) and Ziemann et al. (2008)]. Pharmacological studies show that among others, ICF is most likely to be mediated by the glutamatergic system ( $\mathrm{Zi}$ emann et al., 1998), possibly by the activation of glutamatergic synapses by tRNS. However, no clear evidence was found concerning the cortical origin of ICF, in a recent study in which epidural recording was applied in a conscious subject (Di Lazzaro et al., 2006). The results of this study showed that, despite a significant increase in MEP at ISIs of 10 and $15 \mathrm{~ms}$, there is no evident change in the descending volley. Thus at ISIs of 10 and $15 \mathrm{~ms}$, a small conditioning stimulus can produce clear facilitation of MEPs even though it leads to no detectable change in descending corticospinal activity.

The average MEP decrease observed after mental effort and motor activation are in agreement with previous studies using tDCS (Antal et al., 2007) and paired associative stimulation (PAS) (Stefan et al., 2004). Similarly, a recent study observed that contraction of the FDI muscle during TBS abolished the effects of stimulation on the MEPs (Huang et al., 2008). These results suggest that externally induced neuronal plasticity is highly dependent on the state of the subject during stimulation.

It appears that the tRNS-driven cortical excitability change facilitates the learning process. Previous studies suggest that an excitability enhancement coincides with facilitating the learning process by inducing the strengthening of synapses and inducing long-term potentiation via modifying NMDA-receptor efficacy (RioultPedotti et al., 2000). Regarding studies in the human, this is in line with previous observations of increased activation of the M1 during motor learning tasks (Grafton et al., 1992; Honda et al., 1998), showing that effects of motor training can be improved by cortical excitability enhancements. Additionally, our results describing an increase in corticospinal excitability and facilitating learning with regard to the SRTT more closely resemble those reported by previous studies after anodal tDCS (Nitsche and Paulus, 2000, 2001); even more so, since we applied well proven tDCS parameters such as electrode position, intensity, and stimulation duration.

There is, however, a key difference between tDCS and tRNS. tDCS modifies the transmembrane neuronal potential directly and thus modulates the firing rate of individual neurons (Bindman et al., 1964). In contrast, the stimulation spectrum of tRNS does not possess a DC component. In addition, the physiological control experiment conducted by reversing the electrode position did not influence the characteristic excitability-enhancing 
aftereffect, in contrast to the inhibition that we see with cathodal tDCS (Nitsche and Paulus, 2000). Several physiological mechanisms may underlie the tRNS effects. tRNS, like alternating current stimulation (tACS) (Antal et al., 2008), can possibly interfere with ongoing oscillations and neuronal activity in the brain and thus result in a cortical excitability increase. However, tACS with intensities of $>400 \mu \mathrm{A}$ (Antal et al., 2008) induced a flickering sensation via retinal stimulation, and as a result [at least in the frequency range that we applied $(1-45 \mathrm{~Hz})$ ], we were reluctant to increase the intensity further, at least with the standard reference montage at the forehead close to the retina. Also, the tACS type of monophasic sinusoidal stimulation is more likely to be epileptogenic than that of a random noise waveform. For this reason, we started by using a random noise frequency spectrum with a range of $0.1-640 \mathrm{~Hz}$; the latter frequency is known to represent the high end of physiologically measured human electric brain oscillations (Gobbelé et al., 2000).

We did not make current density calculations of how effectively the high-frequency component of the stimulus is transmitted to the brain. There is, however, sufficient evidence to suggest that the current used here can reach the brain. The bone is the structure with highest resistance, and has to be considered primarily when stimulating the head electrically (Wagner et al., 2008). In fact, high bone resistance was the reason why TMS replaced pulsed electrical stimulation in 1985 (Barker et al., 1985) and thereby could avert painful stimulation. It was found that the bone conductivity on the three orthogonal directions was constant up to $10 \mathrm{kHz}$ (Reddy and Saha, 1984) even above the range of the frequencies used in our study. Distinctly higher frequencies of $50 \mathrm{kHz}$ could still pass through the skull as measured by electrical impedance tomography (Abascal et al., 2008). The dielectric properties of bone was shown to be constant between frequencies of 10 and 100,000 Hz (Gabriel et al., 1996).

A previous study by Yamamoto et al. (2005) used a distinctly lower frequency range $(<2 \mathrm{~Hz})$ in patients with Parkinson's disease (PD). Their method, however, differed from ours in electrode position, stimulation amplitude, duration, and techniques of evaluation. Improved autonomic and motor functions were detected after $24 \mathrm{~h}$ of continuous noisy vestibular electrical stimulation over the bilateral mastoids. The authors hypothesized that in PD patients the input noise ameliorated the impaired neuronal transmission, and the noise itself enhanced weak neuronal signal detection in the sensory system; the phenomenon of stochastic resonance, as shown in several experimental studies (e.g., Moss et al., 2004). Indeed, it has been suggested that noisy electrical fluctuations can boost synaptic signals.

Stochastic resonance may play a role in tRNS, however at much higher frequency ranges. For some years now, oscillations within a frequency range of $80-200 \mathrm{~Hz}$ (ripples) have been associated with plasticity processes (Grenier et al., 2001) and learning (Ponomarenko et al., 2008). Another putative mechanism of tRNS may be activation of sodium channels via rectification by high-frequency stimulation (Bromm, 1968). The postulated tRNS effect begins with the depolarization of a neuronal membrane which causes $\mathrm{Na}^{+}$channels to open. This allows an influx of $\mathrm{Na}^{+}$ions to flow down the concentration gradient and increases membrane depolarization. If the $\mathrm{Na}^{+}$entry is insufficient, there is no regenerative depolarization and thus no action potential, just the "local response." The repolarization occurs passively over a longer period of time compared with the duration of $\mathrm{Na}^{+}$ion entry. If stimulation is repeated, the $\mathrm{Na}^{+}$channels can reopen and induce a second $\mathrm{Na}^{+}$ion influx, which depolarizes the membrane further, heightening the effect of the preceding depolarization. The $\mathrm{Na}^{+}$channels then close, and after repolarization can be reopened by succeeding depolarizations. Indeed, recently it was shown that repetitive extracellular highfrequency stimulation in cultured rat neurons activated an inward sodium current, which gave rise to a weak depolarization of the cell membrane (Schoen and Fromherz, 2008). Although the time integral of the stimulating current used in a voltage clamp study was zero, the average membrane potential was shifted in the direction of depolarization. The resulting depolarization was understood to be the result of the nonlinearity of the sodium current-voltage input during subthreshold excitation. Since we used a symmetric high-frequency stimulation, this nonlinearity could be the reason for the excitatory effects we have seen with tRNS. Interestingly, the effect of tRNS increased with time after stimulation. Effects induced by "repetitive activation of $\mathrm{Na}^{+}$channels by weak capacitive currents" studied by Schoen and Fromherz (2008) also increase with stimulation time, however within a much shorter time range $(<1 \mathrm{~s})$. On the other hand, continuous opening of $\mathrm{Na}^{+}$channels would lead to membrane depolarization, from which we can assume from previous tDCS studies that a time range of $>3$ min may lead to LTP-like mechanisms. However, the neuronal membrane is a more intricate structure and possesses numerous voltage-gated ion channels and is subject to simultaneous influxes of ionic currents $\left(\mathrm{Ca}^{2+}, \mathrm{K}^{+}\right.$, etc). Indeed, because the membrane is encumbered with multiple voltagegated channels, that are "nonlinear," the aforementioned induced changes in membrane fluctuation can be amplified. In summary, a pure DC stimulus can open $\mathrm{Na}^{+}$channels just once, whereas repeated pulses (tRNS) can induce multiple ionic influxes, and achieve substantially heightened effects. The interval at which the pulses are repeated must be short and relates to the time constant of the nerve membrane.

Thus, finally, the neuroplastic effects of tRNS could be analogous to anodal tDCS aftereffects, but with clear advantages. tRNS can circumvent problems that can arise by stimulating a folded cortex with anodal stimulation, since on one side of the gyrus wall current orientation induces excitation, while on the opposite side of the gyrus, it will inevitably induce inhibition. When using tRNS only excitatory aftereffects are observable. Also "tangential" stimulation of nerve cells now appears to be possible with tRNS. Within a "tangential" DC electric field applied to a symmetrical dendritic arbor, currents on both sides would cancel each other at the axon hill. In the case of a rectifying depolarization using a fast oscillating field, the cell would be depolarized regardless of current flow orientation. Safety concerns are probably lessened than in the case of tDCS. Several anecdotal, but so-far-unpublished, reports have described small skin burns after tDCS. In general, nonpolarizing currents seem to be safer than polarizing currents as seen in deep brain stimulation. Here we have not observed any tRNS-induced changes with EEG recordings (see supplemental Table 2, available at www.jneurosci.org as supplemental material). tRNS using $1 \mathrm{~mA}$ was unnoticed in 78 of 80 subjects, compared with a slight skin tingling sensation with tDCS. Thus it appears to have the best blinding potential for controlled studies of currently available methods.

In summary, tRNS allows an unnoticeable and thus painless, selective, focal, noninvasive, and reversible excitability increase of the cortex. Apart from being more economically viable than rTMS its main advantage seems to be the direction insensitivity of the stimulation. It seems to provide a qualitatively new way of producing and interfering with brain plasticity. 


\section{References}

Abascal JF, Arridge SR, Atkinson D, Horesh R, Fabrizi L, De Lucia M, Horesh L, Bayford RH, Holder DS (2008) Use of anisotropic modelling in electrical impedance tomography; description of method and preliminary assessment of utility in imaging brain function in the adult human brain. Neuroimage 43:258-268.

Antal A, Terney D, Poreisz C, Paulus W (2007) Towards unravelling taskrelated modulations of neuroplastic changes induced in the human motor cortex. Eur J Neurosci 26:2687-2691.

Antal A, Boros K, Poreisz C, Chaieb L, Terney D, Paulus W (2008) Comparatively weak after-effects of transcranial alternating current stimulation (tACS) on cortical excitability in humans. Brain Stim 1:97-105.

Barker AT, Jalinous R, Freeston IL (1985) Non-invasive magnetic stimulation of human motor cortex. Lancet 1:1106-1107.

Bindman LJ, Lippold OCJ, Redfearn JWT (1964) The action of brief polarizing currents on the cerebral cortex of the rat (1) during current flow and (2) in the production of long-lasting after-effects. J Physiol 172:369-382.

Bromm B (1968) Die Natrium-Gleichrichtung der unterschwellig erregten Membran in der quantitative Formulierung der Ionentheorie. Pflugers Arch 302:233-244.

Di Lazzaro V, Pilato F, Oliviero A, Dileone M, Saturno E, Mazzone P, Insola A, Profice P, Ranieri F, Capone F, Tonali PA, Rothwell JC (2006) Origin of facilitation of motor-evoked potentials after paired magnetic stimulation: direct recording of epidural activity in conscious humans. J Neurophysiol 96:1765-1771.

Fink GR, Frackowiak RS, Pietrzyk U, Passingham RE (1997) Multiple nonprimary motor areas in the human cortex. J Neurophysiol 77:2164-2174.

Gabriel S, Lau RW, Gabriel C (1996) The dielectric properties of biological tissues: III. Parametric models for the dielectric spectrum of tissues. Phys Med Biol 41:2251-2269.

Gobbelé R, Waberski TD, Kuelkens S, Sturm W, Curio G, Buchner H (2000) Thalamic and cortical high-frequency $(600 \mathrm{~Hz})$ somatosensory-evoked potential (SEP) components are modulated by slight arousal changes in awake subjects. Exp. Brain Res 133:506-513.

Grafton ST, Mazziotta JC, Presty S, Friston KJ, Frackowiak RS, Phelps ME (1992) Functional anatomy of human procedural learning determined with regional cerebral blood flow and PET. J Neurosci 12:2542-2548.

Grenier F, Timofeev I, Steriade M (2001) Focal synchronization of ripples $(80-200 \mathrm{~Hz})$ in neocortex and their neuronal correlates. J Neurophysiol 86:1884-1898.

Honda M, Deiber MP, Ibáñez V, Pascual-Leone A, Zhuang P, Hallett M (1998) Dynamic cortical involvement in implicit and explicit motor sequence learning. A PET study. Brain 121:2159-2173.

Huang YZ, Edwards MJ, Rounis E, Bhatia KP, Rothwell JC (2005) Theta burst stimulation of the human motor cortex. Neuron 45:201-206.

Huang YZ, Rothwell JC, Edwards MJ, Chen RS (2008) Effect of physiological activity on an NMDA-dependent form of cortical plasticity in human. Cereb Cortex 18:563-570.

Kujirai T, Caramia MD, Rothwell JC, Day BL, Thompson PD, Ferbert A, Wroe S, Asselman P, Marsden CD (1993) Corticocortical inhibition in human motor cortex. J Physiol 471:501-519.

Moss F, Ward LM, Sannita WG (2004) Stochastic resonance and sensory information processing: a tutorial and review of application. Clin Neurophysiol 115:267-281.

Münchau A, Bloem BR, Irlbacher K, Trimble MR, Rothwell JC (2002) Functional connectivity of human premotor and motor cortex explored with repetitive transcranial magnetic stimulation. J Neurosci 22:554-561.
Nissen MJ, Bullemer P (1987) Attentional requirements of learning: evidence from performance measures. Cognit Psychol 19:1-32.

Nitsche MA, Paulus W (2000) Excitability changes induced in the human motor cortex by weak transcranial direct current stimulation. J Physiol 527:633-639.

Nitsche MA, Paulus W (2001) Sustained excitability elevations induced by transcranial DC motor cortex stimulation in humans. Neurology 57:1899-1901

Nitsche MA, Liebetanz D, Lang N, Antal A, Tergau F, Paulus W (2003) Safety criteria for transcranial direct current stimulation (tDCS) in humans. Clin Neurophysiol 114:2220-2222; author reply 2222-2223.

Oldfield RC (1971) The assessment and analysis of handedness: the Edinburgh inventory. Neuropsychologia 9:97-113.

Paulus W, Classen J, Cohen LG, Large CH, Di Lazzaro V, Nitsche MA, Pascual-Leone A, Rosenow F, Rothwell JC, Ziemann U (2008) State of the art: pharmacologic effects on cortical excitability measures tested by transcranial magnetic stimulation. Brain Stim 1:151-163.

Ponomarenko AA, Li JS, Korotkova TM, Huston JP, Haas HL (2008) Frequency of network synchronization in the hippocampus marks learning. Eur J Neurosci 27:3035-3042.

Reddy GN, Saha S (1984) Electrical and dielectrical properties of wet bone as a function of frequency. IEEE Trans Biomed Eng 31:296-303.

Rioult-Pedotti MS, Friedman D, Donoghue JP (2000) Learning-induced LTP in neocortex. Science 290:533-536.

Rothwell JC, Hallett M, Berardelli A, Eisen A, Rossini P, Paulus W (1999) Magnetic stimulation: motor evoked potentials: the International Federation of Clinical Neurophysiology. Electroencephalogr Clin Neurophysiol Suppl 52:97-103.

Schoen I, Fromherz P (2008) Extracellular stimulation of mammalian neurons through repetitive activation of $\mathrm{Na}^{+}$channels by weak capacitive currents on a silicon chip. J Neurophysiol 100:346-357.

Stefan K, Wycislo M, Classen J (2004) Modulation of associative human motor cortical plasticity by attention. J Neurophysiol 92:66-72.

Steinhoff BJ, Tumani H, Otto M, Mursch K, Wiltfang J, Herrendorf G, Bittermann HJ, Felgenhauer K, Paulus W, Markakis E (1999) Cisternal S100 protein and neuron-specific enolase are elevated and site-specific markers in intractable temporal lobe epilepsy. Epilepsy Res 36:75-82.

Valls-Solé J, Pascual-Leone A, Wassermann EM, Hallett M (1992) Human motor evoked responses to paired transcranial magnetic stimuli. Electroencephalogr Clin Neurophysiol 85:355-364.

Wagner T, Eden U, Fregni F, Valero-Cabre A, Ramos-Estebanez C, PronioStelluto V, Grodzinsky A, Zahn M, Pascual-Leone A (2008) Transcranial magnetic stimulation and brain atrophy: a computer-based human brain model study. Exp Brain Res 186:539-550.

Wassermann EM (1998) Risk and safety of repetitive transcranial magnetic stimulation: report and suggested guidelines from the International Workshop on the Safety of Repetitive Transcranial Magnetic Stimulation, June 5-7, 1996. Electroencephalogr Clin Neurophysiol 108:1-16.

Yamamoto Y, Struzik ZR, Soma R, Ohashi K, Kwak S (2005) Noisy vestibular stimulation improves autonomic and motor responsiveness in central neuro-degenerative disorders. Ann Neurol 58:175-181.

Ziemann U, Chen R, Cohen LG, Hallett M (1998) Dextromethorphan decreases the excitability of the human motor cortex. Neurology 51:1320-1324.

Ziemann U, Paulus W, Nitsche MA, Pascual-Leone A, Byblow WD, Berardelli A, Siebner HR, Classen J, Cohen LG, Rothwell JC (2008) Consensus: motor cortex plasticity protocols. Brain Stim 1:164-182. 